\title{
The poles and growth of solutions of systems of complex difference equations
}

\author{
Hua Wang ${ }^{*}$, Hong-Yan Xu and Bing-Xiang Liu
}

${ }^{*}$ Correspondence:

hhhlucy2012@126.com

Department of Informatics and Engineering, Jingdezhen Ceramic

Institute, Jingdezhen, Jiangxi

333403, China

\begin{abstract}
In view of the Nevanlinna theory, we study the growth and poles of solutions of some classes of systems of complex difference equations and obtain some interesting results such as the lower bounds for Nevanlinna lower order, a counting function of poles and maximum modulus for solutions of such systems. They extend some results concerning functional equations to the systems of functional equations in the fields of complex equations.
\end{abstract}

MSC: 39A50; 30D35

Keywords: growth; difference equation; pole

\section{Introduction and main results}

The purpose of this paper is to study some properties of the poles and growth of meromorphic solutions of the systems of complex difference equations. The fundamental results and the standard notations of the Nevanlinna value distribution theory of meromorphic functions are used (see [1-3]). In this paper, a meromorphic function means being meromorphic in the whole complex plane $\mathbb{C}$; for a meromorphic function $f, S(r, f)$ denotes any quantity satisfying $S(r, f)=o(T(r, f))$ for all $r$ outside a possible exceptional set $E$ of finite logarithmic measure $\lim _{r \rightarrow \infty} \int_{[1, r) \cap E} \frac{d t}{t}<\infty$, and a meromorphic function $a(z)$ is called a small function with respect to $f$ if $T(r, a(z))=S(r, f)=o(T(r, f))$.

In 1980, Shimomura [4] and Yanagihara [5] studied some existence of solutions of difference equations and obtained some theorems as follows.

Theorem 1.1 (see [4, Theorem 2.5]) For any non-constant polynomial $P(y)$, the difference equation

$$
y(z+1)=P(y(z))
$$

has a non-trivial entire solution.

Theorem 1.2 (see [5, Corollary 6]) For any non-constant rational function $R(y)$, the difference equation

$$
y(z+1)=R(y(z))
$$

has a non-trivial meromorphic solution in the complex plane.

\section{Springer}

(0) 2013 Wang et al.; licensee Springer. This is an Open Access article distributed under the terms of the Creative Commons Attribution License (http://creativecommons.org/licenses/by/2.0), which permits unrestricted use, distribution, and reproduction in any medium, provided the original work is properly cited. 
It was proposed that the existence of sufficiently many meromorphic solutions of finite order would be a strong indicator of integrability of an equation (see [6-8]).

In 2000, Ablowitz, Halburd and Herbst [6] studied some classes of complex difference equations

$$
\begin{gathered}
f(z+1)+f(z-1)=\frac{a_{0}(z)+a_{1}(z) f+\cdots+a_{p}(z) f^{p}}{b_{0}(z)+b_{1}(z) f+\cdots+b_{q}(z) f^{q}}, \\
f(z+1) f(z-1)=\frac{a_{0}(z)+a_{1}(z) f+\cdots+a_{p}(z) f^{p}}{b_{0}(z)+b_{1}(z) f+\cdots+b_{q}(z) f^{q}},
\end{gathered}
$$

where the coefficients are meromorphic functions, and obtained the following results.

Theorem 1.3 (see [6]) If difference equation (1) (or (2)) with polynomial coefficients $a_{i}(z)$, $b_{i}(z)$ admits a transcendental meromorphic solution of finite order, then $d=\max \{p, q\} \leq 2$.

In 2001, Heittokangas et al. [9] further investigated some complex difference equations which are similar to (1) and (2) and obtained the following results which are improvements of Theorems 1.1 and 1.2.

Theorem 1.4 (see [9, Proposition 8 and Proposition 9]) Let $c_{1}, \ldots, c_{n} \in \mathbb{C} \backslash\{0\}$. If the equations

$$
\begin{aligned}
& \sum_{i=1}^{n} f\left(z+c_{i}\right)=R(z, f(z)), \quad \prod_{i=1}^{n} f\left(z+c_{i}\right)=R(z, f(z)), \\
& R(z, f(z)):=\frac{P(z, f(z))}{Q(z, f(z))}=\frac{a_{0}(z)+a_{1}(z) f+\cdots+a_{s}(z) f^{s}}{b_{0}(z)+b_{1}(z) f+\cdots+b_{t}(z) f^{t}}
\end{aligned}
$$

with rational coefficients $a_{i}(z), b_{i}(z)$ admit a transcendental meromorphic solution of finite order, then $d=\max \{s, t\} \leq n$.

In the same paper, some results of the lower bound for the characteristic functions, poles and maximum modulus of transcendental meromorphic solutions of some complex difference equations are obtained as follows.

Theorem 1.5 (see [9, Theorem 10]) Let $c_{1}, \ldots, c_{n} \in C \backslash\{0\}$ and let $m \geq 2$. Suppose $y$ is a transcendental meromorphic solution of the difference equation

$$
\sum_{i=1}^{n} a_{i}(z) y\left(z+c_{i}\right)=\sum_{i=0}^{m} b_{i}(z) y(z)^{i}
$$

with rational coefficients $a_{i}(z), b_{i}(z)$. Denote $C:=\max \left\{\left|c_{1}\right|, \ldots,\left|c_{n}\right|\right\}$.

(1) If $y$ is entire or has finitely many poles, then there exist constants $K>0$ and $r_{0}>0$ such that

$$
\log M(r, y) \geq K m^{r / C}
$$

holds for all $r \geq r_{0}$. 
(2) If $y$ has infinitely many poles, then there exist constants $K>0$ and $r_{0}>0$ such that

$$
n(r, y) \geq K m^{r / c}
$$

holds for all $r \geq r_{0}$.

Theorem 1.6 (see [9, Theorem 11]) Let $c_{1}, \ldots, c_{n} \in \mathbb{C} \backslash\{0\}$ and suppose that $y$ is a nonrational meromorphic solution of

$$
\sum_{i=1}^{n} d_{i}(z) y\left(z+c_{i}\right)=\frac{a_{0}(z)+a_{1}(z) f+\cdots+a_{p}(z) f^{p}}{b_{0}(z)+b_{1}(z) f+\cdots+b_{q}(z) f^{q}}
$$

where all coefficients in (3) are of growth $o(T(r, y))$ without an exceptional set as $r \rightarrow \infty$, and $d_{i}$ 's are non-vanishing. If $d=\max \{p, q\}>n$, then for any $\varepsilon(0<\varepsilon<(d-n) /(d+n))$, there exists an $r_{0}>0$ such that

$$
T(r, y) \geq K\left(\frac{d}{n}\left(\frac{1-\varepsilon}{1+\varepsilon}\right)\right)^{r / C}
$$

for all $r \geq r_{0}$, where $C:=\max \left\{\left|c_{1}\right|, \ldots,\left|c_{n}\right|\right\}$ and $K>0$ is a constant.

Recently, a number of papers have focused on difference equations, difference product and $q$-difference in the complex plane $\mathbb{C}$, and considerable attention has been paid to the growth of solutions of difference equations, value distribution and uniqueness of differences analogues of Nevanlinna's theory [4, 6-25].

In 2012, Gao [14-16] also investigated the growth and existence of meromorphic solutions of some systems of complex difference equations and obtained some existence theorems and estimates on the proximity function and the counting function of solutions of some systems of complex difference equations.

Inspired by the ideas of Refs. [14-16] and Ref. [9], we investigate the growth and poles of meromorphic solutions of some systems of complex difference equations and obtain the following results.

Theorem 1.7 Suppose that $\left(f_{1}, f_{2}\right)$ is a transcendental meromorphic solution of a system of difference equations of the form

$$
\left\{\begin{array}{l}
\sum_{j=1}^{n} a_{j}^{1}(z) f_{1}\left(z+c_{j}\right)=\sum_{i=0}^{d_{1}} b_{i}^{1}(z) f_{2}(z)^{i}, \\
\sum_{j=1}^{n} a_{j}^{2}(z) f_{2}\left(z+c_{j}\right)=\sum_{i=0}^{d_{2}} b_{i}^{2}(z) f_{1}(z)^{i},
\end{array}\right.
$$

where $d_{1} d_{2} \geq 2$ and the coefficients $a_{j}^{t}(z), b_{i}^{t}(z)(t=1,2)$ are rational functions. Denote $C:=\max \left\{\left|c_{1}\right|, \ldots,\left|c_{n}\right|\right\}$. If $f_{t}(t=1,2)$ are entire or have finitely many poles, then there exist constants $K_{t}>0(t=1,2)$ and $r_{0}>0$ such that for all $r \geq r_{0}$

$$
\log M\left(r, f_{t}\right) \geq K_{t}\left(d_{1} d_{2}\right)^{r /(2 C)}, \quad t=1,2
$$


Theorem 1.8 Suppose that $\left(f_{1}, f_{2}\right)$ is a transcendental meromorphic solution of a system of difference equations of the form

$$
\left\{\begin{array}{l}
\sum_{j=1}^{n_{1}} a_{j}^{1}(z) f_{1}\left(z+c_{j}\right)=R_{2}\left(z, f_{2}(z)\right)=\frac{P_{2}\left(z, f_{2}(z)\right)}{Q_{2}\left(z f_{2}(z)\right)} \\
\sum_{j=1}^{n_{2}} a_{j}^{2}(z) f_{2}\left(z+c_{j}\right)=R_{1}\left(z, f_{1}(z)\right)=\frac{P_{1}\left(z, f_{1}(z)\right)}{Q_{1}\left(z, f_{1}(z)\right)}
\end{array}\right.
$$

where the coefficients $a_{j}^{t}(z), t=1,2$ are rational functions, and $P_{t}, Q_{t}$ are relatively prime polynomials in $f_{t}$ over the field of rational functions satisfying $p_{t}=\operatorname{deg}_{f_{t}} P_{t}, l_{t}=\operatorname{deg}_{f_{t}} Q_{t}$, $d_{t}=p_{t}-l_{t} \geq 2, t=1,2$. Denote $C:=\max \left\{\left|c_{1}\right|, \ldots,\left|c_{n}\right|\right\}$. If $f_{t}$ have infinitely many poles, then for sufficiently large $r$,

$$
n\left(r, f_{t}\right) \geq K_{t}\left(d_{1} d_{2}\right)^{r /(2 C)}, \quad t=1,2 .
$$

Remark 1.1 Since system (4) is a particular case of system (5), from the conclusions of Theorem 1.8, we can get the following result.

Under the assumptions of Theorem 1.7, if $f_{t}(t=1,2)$ have infinitely many poles, then there exist constants $K_{t}>0(t=1,2)$ and $r_{0}>0$ such that for all $r \geq r_{0}$,

$$
n\left(r, f_{t}\right) \geq K_{t}\left(d_{1} d_{2}\right)^{r /(2 C)}, \quad t=1,2
$$

Theorem 1.9 Suppose that $\left(f_{1}, f_{2}\right)$ is a transcendental meromorphic solution of a system of complex difference equations of the form

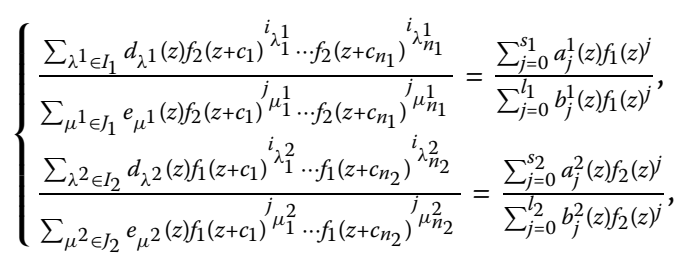

where $I_{t}=\left\{i_{\lambda_{1}^{t}}, i_{\lambda_{2}^{t}}, \ldots, i_{\lambda_{n_{t}}^{t}}\right\}, J_{t}=\left\{j_{\mu_{1}^{t}}, j_{\mu_{2}^{t}}, \ldots, j_{\mu_{n_{t}}^{t}}\right\}$ are finite index sets satisfying

$$
\max _{\lambda^{t}, \mu^{t}}\left\{i_{\lambda_{1}^{t}}+i_{\lambda_{2}^{t}}+\cdots+i_{\lambda_{n_{t}}^{t}}, j_{\mu_{1}^{t}}+j_{\mu_{2}^{t}}+\cdots+j_{\mu_{n t}^{t}}\right\}=\sigma_{t}, \quad t=1,2,
$$

$d_{t}=\max \left\{s_{t}, l_{t}\right\} \geq 2, t=1,2, n_{1}, n_{2} \in \mathbb{N}_{+}$and all coefficients of (6) are of growth $o\left(T\left(r, f_{1}\right)\right)$, $o\left(T\left(r, f_{2}\right)\right)$ without an exceptional set. Denote $C:=\max \left\{\left|c_{1}\right|, \ldots,\left|c_{n}\right|\right\}$. If $d_{1} d_{2}>4 n_{1} n_{2} \sigma_{1} \sigma_{2}$, then for any $\varepsilon$ satisfying

$$
0<\varepsilon<\frac{\sqrt{d_{1} d_{2}}-\sqrt{4 n_{1} n_{2} \sigma_{1} \sigma_{2}}}{\sqrt{d_{1} d_{2}}+\sqrt{4 n_{1} n_{2} \sigma_{1} \sigma_{2}}}
$$

there exist constants $r_{0}>0$ and $K_{t}>0(t=1,2)$ for all $r>r_{0}$,

$$
T\left(r, f_{t}\right) \geq K_{t}\left(\frac{d_{1} d_{2}}{4 n_{1} n_{2} \sigma_{1} \sigma_{2}}\left(\frac{1-\varepsilon}{1+\varepsilon}\right)^{2}\right)^{r /(2 C)}, \quad t=1,2 .
$$

Theorem 1.10 Suppose that all coefficients in (6) are of growth $S\left(r, f_{1}\right), S\left(r, f_{2}\right)$ and that all the other assumptions of Theorem 1.9 hold. Then $\mu\left(f_{t}\right)=\infty(t=1,2)$. 


\section{Some lemmas}

Lemma 2.1 (Valiron-Mohon'ko [21]) Let $f(z)$ be a meromorphic function. Then for all irreducible rational functions in $f$,

$$
R(z, f(z))=\frac{\sum_{i=0}^{m} a_{i}(z) f(z)^{i}}{\sum_{j=0}^{n} b_{j}(z) f(z)^{j}}
$$

with meromorphic coefficients $a_{i}(z), b_{j}(z)$, the characteristic function of $R(z, f(z))$ satisfies

$$
T(r, R(z, f(z)))=d T(r, f)+O(\Psi(r))
$$

where $d=\max \{m, n\}$ and $\Psi(r)=\max _{i, j}\left\{T\left(r, a_{i}\right), T\left(r, b_{j}\right)\right\}$.

Lemma 2.2 (see [25]) Let $f_{1}, f_{2}, \ldots, f_{n}$ be meromorphic functions. Then

$$
T\left(r, \sum_{\lambda \in I} f_{1}^{i_{\lambda_{1}}} f_{2}^{i_{\lambda_{2}}} \cdots f_{n}^{i_{\lambda_{n}}}\right) \leq \sigma \sum_{i=1}^{n} T\left(r, f_{i}\right)+\log s
$$

where $I=\left\{i_{\lambda_{1}}, i_{\lambda_{2}}, \ldots, i_{\lambda_{n}}\right\}$ is an index set consisting of s elements, and $\sigma=\max _{\lambda \in I}\left\{i_{\lambda_{1}}+i_{\lambda_{2}}+\right.$ $\left.\cdots+i_{\lambda_{n}}\right\}$.

Lemma 2.3 (see [21]) Let $g:(0,+\infty) \rightarrow \mathbb{R}, h:(0,+\infty) \rightarrow \mathbb{R}$ be monotone increasing functions such that $g(r) \leq h(r)$ outside of an exceptional set of finite logarithmic measure. Then for any $\alpha>1$, there exists $r_{0}>0$ such that $g(r) \leq h(\alpha r)$ for all $r>r_{0}$.

Lemma 2.4 (see [6, Lemma 1]) Given $\epsilon>0$ and a meromorphic function $y$, the Nevanlinna characteristic function $T$ satisfies

$$
T(r, y(z \pm 1)) \leq(1+\varepsilon) T(r+1, y(z))+\kappa
$$

for all $r \geq 1 / \epsilon$, for some constant $\kappa$.

\section{The proof of Theorem 1.7}

Since the coefficients $a_{j}^{t}(z), b_{i}^{t}(z)(t=1,2)$ are rational functions, we can rewrite (4) as

$$
\left\{\begin{array}{l}
\sum_{j=1}^{n} A_{j}^{1}(z) f_{1}\left(z+c_{j}\right)=\sum_{i=0}^{d_{1}} B_{i}^{1}(z) f_{2}(z)^{i} \\
\sum_{j=1}^{n} A_{j}^{2}(z) f_{2}\left(z+c_{j}\right)=\sum_{i=0}^{d_{2}} B_{i}^{2}(z) f_{1}(z)^{i}
\end{array}\right.
$$

where the coefficients $A_{j}^{t}(z), B_{i}^{t}(z)(t=1,2)$ are polynomials.

Next, two cases will be considered as follows.

Case 1. Since $\left(f_{1}, f_{2}\right)$ is a transcendental solution of system (4) or (7) and $f_{t}(t=1,2)$ are entire, set $p_{i}^{t}=\operatorname{deg} A_{j}^{t}(j=1,2, \ldots, n), q_{i}^{t}=\operatorname{deg} B_{i}^{t}\left(i=0,1, \ldots, d_{i}\right), t=1,2$, taking $m_{t}=$ $\max \left\{p_{1}^{t}, \ldots, p_{n}^{t}\right\}+1$, we have that

$$
\left\{\begin{array}{l}
M\left(r, \sum_{i=0}^{d_{1}} B_{i}^{1}(z) f_{2}(z)^{i}\right)=M\left(r, \sum_{j=1}^{n} A_{j}^{1}(z) f_{1}\left(z+c_{j}\right)\right) \leq n r^{m_{1}} M\left(r+C, f_{1}\right), \\
M\left(r, \sum_{i=0}^{d_{2}} B_{i}^{2}(z) f_{1}(z)^{i}\right)=M\left(r, \sum_{j=1}^{n} A_{j}^{2}(z) f_{2}\left(z+c_{j}\right)\right) \leq n r^{m_{2}} M\left(r+C, f_{2}\right),
\end{array}\right.
$$


when $r$ is sufficiently large. Since $f_{t}$ are transcendental entire functions and $B_{i}^{t}$ ( $i=$ $\left.0,1, \ldots, d_{t} ; t=1,2\right)$ are polynomials, we have $M\left(r, \sum_{i=0}^{d_{1}-1} B_{i}^{1} f_{2}(z)^{i}\right)=o\left(M\left(r, f_{2}(z)^{d_{1}}\right)\right)$ and $M\left(r, \sum_{i=0}^{d_{2}-1} B_{i}^{2} f_{1}(z)^{i}\right)=o\left(M\left(r, f_{1}(z)^{d_{2}}\right)\right)$. Thus, for sufficiently large $r$, we have

$$
\left\{\begin{array}{l}
M\left(r, \sum_{i=0}^{d_{1}} B_{i}^{1}(z) f_{2}(z)^{i}\right) \geq \frac{1}{2} M\left(r, B_{d_{1}}^{1} f_{2}(z)^{d_{1}}\right), \\
M\left(r, \sum_{i=0}^{d_{2}} B_{i}^{2}(z) f_{1}(z)^{i}\right) \geq \frac{1}{2} M\left(r, B_{d_{2}}^{2} f_{1}(z)^{d_{2}}\right) .
\end{array}\right.
$$

From (8) and (9), we have

$$
\left\{\begin{array}{l}
\log M\left(r+C, f_{1}\right) \geq d_{1} \log M\left(r, f_{2}\right)+g_{1}(r), \\
\log M\left(r+C, f_{2}\right) \geq d_{2} \log M\left(r, f_{1}\right)+g_{2}(r),
\end{array}\right.
$$

where $\left|g_{t}(r)\right|<K_{t} \log r, t=1,2$ for some constants $K_{t}>0$ and sufficiently large $r$. From (10), for sufficiently large $r$, we have

$$
\log M\left(r+2 C, f_{1}\right) \geq d_{1} d_{2} \log M\left(r, f_{1}\right)+g_{1}(r+C)+d_{1} g_{2}(r) .
$$

Iterating (11), we have

$$
\log M\left(r+2 k C, f_{1}\right) \geq\left(d_{1} d_{2}\right)^{k} \log M\left(r, f_{1}\right)+E_{k}^{1}(r)+E_{k}^{2}(r) \quad(k \in \mathbb{N}),
$$

where

$$
\begin{aligned}
\left|E_{k}^{1}(r)\right| & =\left|\left(d_{1} d_{2}\right)^{k-1} g_{1}(r+C)+\left(d_{1} d_{2}\right)^{k-2} g_{1}(r+3 C)+\cdots+g_{1}(r+(2 k-1) C)\right| \\
& \leq K_{1}\left(d_{1} d_{2}\right)^{k-1} \sum_{j=1}^{k} \frac{\log [r+(2 j-1) C]}{\left(d_{1} d_{2}\right)^{j}} \\
& \leq K_{1}\left(d_{1} d_{2}\right)^{k-1} \sum_{j=1}^{\infty} \frac{\log [r+(2 j-1) C]}{\left(d_{1} d_{2}\right)^{j-1}},
\end{aligned}
$$

and

$$
\begin{aligned}
\left|E_{k}^{2}(r)\right| & =\left|d_{1}\left(d_{1} d_{2}\right)^{k-1} g_{2}(r)+d_{1}\left(d_{1} d_{2}\right)^{k-2} g_{2}(r+2 C)+\cdots+d_{1} g_{2}(r+2(k-1) C)\right| \\
& \leq K_{2} d_{1}\left(d_{1} d_{2}\right)^{k-1} \sum_{j=1}^{k} \frac{\log [r+2(j-1) C]}{\left(d_{1} d_{2}\right)^{j-1}} \\
& \leq K_{2} d_{1}\left(d_{1} d_{2}\right)^{k-1} \sum_{j=1}^{\infty} \frac{\log [r+2(j-1) C]}{\left(d_{1} d_{2}\right)^{j-1}} .
\end{aligned}
$$

Since $\log [r+k C] \leq \log r \times \log k C$ for sufficiently large $r$ and $k$, and since $d_{1} d_{2} \geq 2$, we know that the series $\sum_{j=1}^{\infty} \frac{\log [r+(2 j-1) C]}{\left(d_{1} d_{2}\right)^{j-1}}$ and $\sum_{j=1}^{\infty} \frac{\log [r+2(j-1) C]}{\left(d_{1} d_{2}\right)^{j-1}}$ are convergent. Thus, for sufficiently large $r$, we have

$$
\left|E_{k}^{t}(r)\right| \leq K_{t}^{\prime}\left(d_{1} d_{2}\right)^{k} \log r, \quad t=1,2,
$$


where $K_{t}^{\prime}>0(t=1,2)$ are some constants. Since $f_{1}$ is a transcendental entire function, for sufficiently large $r$, we have

$$
\log M\left(r, f_{1}\right) \geq 3 K^{\prime} \log r
$$

where $K^{\prime}>\max \left\{K_{1}^{\prime}, K_{2}^{\prime}\right\}$. Hence, from (12)-(14), there exists $r_{0} \geq e$ such that for $r \geq r_{0}$, we have

$$
\log M\left(r+2 k C, f_{1}\right) \geq K^{\prime}\left(d_{1} d_{2}\right)^{k} \log r .
$$

Choosing $r \in\left[r_{0}, r_{0}+C\right)$ and letting $k \rightarrow \infty$ for each choice of $r$, and for each sufficiently large $R:=r+2 k C \geq R_{0}:=r_{0}+C$, we have

$$
R \in\left[r_{0}+2 k C, r_{0}+(2 k+1) C\right), \quad \text { i.e. } k>\frac{R-r_{0}-C}{2 C} \text {. }
$$

From (15) and (16), we have

$$
\log M\left(R, f_{1}\right) \geq \log M\left(r_{0}+2 k C, f_{1}\right) \geq K^{\prime}\left(d_{1} d_{2}\right)^{k} \log r_{0} \geq K^{\prime \prime}\left(d_{1} d_{2}\right)^{R /(2 C)},
$$

where $K^{\prime \prime}=K^{\prime}\left(d_{1} d_{2}\right)^{\frac{-r_{0}-C}{2 C}} \log r_{0}$.

By using the same argument as above, we can get that there exist constants $K>0$ and $r_{0}>0$ such that for all $R \geq r_{0}$,

$$
\log M\left(R, f_{2}\right) \geq K\left(d_{1} d_{2}\right)^{R /(2 C)}
$$

Case 2. Suppose that $\left(f_{1}, f_{2}\right)$ is a solution of system $(4)$ and $f_{t}(t=1,2)$ are meromorphic functions with finitely many poles. Then there exist polynomials $P_{t}(z)$ such that $g_{t}(z)=P_{t}(z) f_{t}(z)(t=1,2)$ are entire functions. Substituting $f_{t}(z)=\frac{g_{t}(z)}{P_{t}(z)}$ into $(7)$ and again multiplying away the denominators, we can get a system similar to (7). By using the same argument as above, we can obtain that for sufficiently large $r \geq r_{1} \geq r_{0}$,

$$
\log M\left(r, f_{t}\right)=\log M\left(r, g_{t}\right)+\log M\left(r, \frac{1}{P_{t}(z)}\right) \geq\left(K_{t}^{\prime \prime}-\varepsilon\right)\left(d_{1} d_{2}\right)^{r /(2 C)} \geq K_{t}^{\prime \prime \prime}\left(d_{1} d_{2}\right)^{r /(2 C)}
$$

where $K_{t}^{\prime \prime \prime}(>0)(t=1,2)$ are some constants.

From Case 1 and Case 2, this completes the proof of Theorem 1.7.

\section{The proof of Theorem 1.8}

Suppose that $\left(f_{1}, f_{2}\right)$ is a solution of system $(5)$ and $f_{t}(t=1,2)$ are transcendental. Since the coefficients of $P_{t}\left(z, f_{t}(z)\right), Q_{t}\left(z, f_{t}(z)\right)$ are rational functions, we can choose a sufficiently large constant $R(>0)$ such that the coefficients of $P_{t}\left(z, f_{t}(z)\right), Q_{t}\left(z, f_{t}(z)\right)$ have no zeros or poles in $\{z \in \mathbb{C}:|z|>R\}$. Since $f_{t}(t=1,2)$ have infinitely many poles, we can choose a pole $z_{0}$ of $f_{1}$ of multiplicity $\tau \geq 1$ satisfying $\left|z_{0}\right|>R$. Then the right-hand side of the second equation in system (5) has a pole of multiplicity $d_{1} \tau$ at $z_{0}$. Then there exists at least one index $j_{1} \in\left\{1,2, \ldots, n_{2}\right\}$ such that $z_{0}+c_{j_{1}}$ is a pole of $f_{2}$ of multiplicity $\tau_{1}^{\prime} \geq d_{1} \tau$. Replacing $z$ 
by $z_{0}+c_{j_{1}}$ in the first equation of (5), we have

$$
\sum_{j=1}^{n_{1}} a_{j}^{1}\left(z_{0}+c_{j_{1}}\right) f_{1}\left(z_{0}+c_{j_{1}}+c_{j}\right)=R_{2}\left(z_{0}+c_{j_{1}}, f_{2}\left(z_{0}+c_{j_{1}}\right)\right)
$$

We now have two possibilities as follows.

(i) If $z_{0}+c_{j_{1}}$ is a pole or a zero of the coefficients of $R_{2}\left(z, f_{2}(z)\right)$, then this process will be terminated and we can choose another pole $z_{0}$ of $f_{1}$ in the way we did above.

(ii) If $z_{0}+c_{j_{1}}$ is neither a pole nor a zero of the coefficients of $R_{2}\left(z, f_{2}(z)\right)$, thus the righthand side of (18) has a pole of multiplicity $d_{2} \tau_{1}^{\prime}$ at $z_{0}+c_{j_{1}}$, then there exists at least one index $j_{1}^{\prime} \in\left\{1,2, \ldots, n_{1}\right\}$ such that $z_{0}+c_{j_{1}}+c_{j_{1}^{\prime}}$ is a pole of $f_{1}$ of multiplicity $\tau_{1} \geq d_{2} \tau_{1}^{\prime} \geq d_{1} d_{2} \tau$. Replacing $z$ by $z_{0}+c_{j_{1}}+c_{j_{1}^{\prime}}$ in the second equation of (5), we have

$$
\sum_{j=1}^{n_{2}} a_{j}^{2}\left(z_{0}+c_{j_{1}}+c_{j_{1}^{\prime}}\right) f_{2}\left(z_{0}+c_{j_{1}}+c_{j_{1}^{\prime}}+c_{j}\right)=R_{1}\left(z_{0}+c_{j_{1}}+c_{j_{1}^{\prime}}, f_{1}\left(z_{0}+c_{j_{1}}+c_{j_{1}^{\prime}}\right)\right) .
$$

We proceed to follow the step above. Since the coefficients of $R_{t}\left(z, f_{t}(z)\right)$ have finitely many zeros and poles in $\{z \in \mathbb{C}:|z|>R\}$ and $f_{1}$ has infinitely many poles again, we may construct poles $\zeta_{2 k}:=z_{0}+c_{j_{1}}+c_{j_{1}^{\prime}}+c_{j_{2}}+c_{j_{2}^{\prime}}+\cdots+c_{j_{k}}+c_{j_{k}^{\prime}}\left(j_{i} \in\left\{1,2, \ldots, n_{2}\right\}, j_{i}^{\prime} \in\left\{1,2, \ldots, n_{1}\right\}\right.$, $i=1,2, \ldots, k)$ of $f_{1}$ of multiplicity $\tau_{k}$ satisfying $\tau^{k} \geq\left(d_{1} d_{2}\right)^{k} \tau$ as $k \rightarrow \infty, k \in \mathbb{N}$. Since $\left|\zeta_{2 k}\right| \rightarrow$ $\infty$ as $k \rightarrow \infty$, for sufficiently large $k$, say $k \geq k_{0}$ and any $\mathscr{R}_{1} \in\left[\left|z_{0}\right|,\left|z_{0}\right|+C\right)$, we have

$$
\begin{aligned}
\tau\left(d_{1} d_{2}\right)^{k} & \leq \tau\left(1+d_{1} d_{2}+\cdots+\left(d_{1} d_{2}\right)^{k}\right) \leq n\left(\left|\zeta_{2 k}\right|, f_{1}\right) \\
& =n\left(\left|z_{0}\right|+2 k C, f_{1}\right) \leq n\left(\mathscr{R}_{1}+2 k C, f_{1}\right) .
\end{aligned}
$$

If we can choose a pole $z_{1}$ of $f_{2}$ of multiplicity $\tau^{\prime} \geq 1$ satisfying $\left|z_{1}\right|>R$, similar to the above discussion, we can get that for sufficiently large $k$ and any $\mathscr{R}_{2} \in\left[\left|z_{1}\right|,\left|z_{1}\right|+C\right)$,

$$
\tau^{\prime}\left(d_{1} d_{2}\right)^{k} \leq n\left(\left|z_{1}\right|+2 k C, f_{2}\right) \leq n\left(\mathscr{R}_{2}+2 k C, f_{2}\right)
$$

Thus, for each sufficiently large $\mathscr{R}:=\mathscr{R}_{1}+2 k C \geq r_{0}:=\left|z_{0}\right|+2\left(k_{0}+1\right) C$, there exists a $k \in \mathbb{N}$ such that $\mathscr{R} \in\left[\left|z_{0}\right|+2 k C,\left|z_{0}\right|+(2 k+1) C\right)\left(\right.$ or $\left.\mathscr{R} \in\left[\left|z_{1}\right|+2 k C,\left|z_{1}\right|+(2 k+1) C\right)\right)$, by using the same method as in the proof of Theorem 1.7, from (19) (or (20)), we have

$$
n\left(\mathscr{R}, f_{1}\right) \geq \tau\left(d_{1} d_{2}\right)^{k} \geq \tau\left(d_{1} d_{2}\right)^{\frac{\mathscr{R}-\left|z_{0}\right|-C}{2 C}} \geq K_{1}\left(d_{1} d_{2}\right)^{\mathscr{R} /(2 C)},
$$

or

$$
n\left(\mathscr{R}, f_{2}\right) \geq \tau^{\prime}\left(d_{1} d_{2}\right)^{k} \geq \tau\left(d_{1} d_{2}\right)^{\frac{\mathscr{R}-\left|z_{1}\right|-C}{2 C}} \geq K_{2}\left(d_{1} d_{2}\right)^{\mathscr{R} /(2 C)},
$$

where $K_{1}=\tau\left(d_{1} d_{2}\right)^{\frac{-\left|z_{0}\right|-C}{2 C}}$ and $K_{2}=\tau^{\prime}\left(d_{1} d_{2}\right)^{\frac{-\left|z_{1}\right|-C}{2 C}}$.

Thus, from (21) and (22), this completes the proof of Theorem 1.8.

\section{The proof of Theorem 1.9}

From the assumptions of Theorem 1.9 and $f_{t}(t=1,2)$ are transcendental, applying Lemma 2.1 and Lemma 2.2 for (6) and by Lemma 2.4, for any given $\varepsilon(0<\varepsilon<$ 
$\left.\frac{\sqrt{d_{1} d_{2}}-\sqrt{4 n_{1} n_{2} \sigma_{1} \sigma_{2}}}{\sqrt{d_{1} d_{2}}+\sqrt{4 n_{1} n_{2} \sigma_{1} \sigma_{2}}}\right)$ and all $r \geq r_{0} \geq \frac{1}{\varepsilon}$, we have

$$
\left\{\begin{aligned}
d_{1}(1-\varepsilon) T\left(r, f_{1}\right) & \leq d_{1} T\left(r, f_{1}\right)+o\left(T\left(r, f_{1}\right)\right) \leq 2 \sigma_{1} \sum_{j=1}^{n_{1}} T\left(r+C, f_{2}\right)+o\left(T\left(r, f_{2}\right)\right) \\
& \leq 2 n_{1} \sigma_{1}(1+\varepsilon) T\left(r+C, f_{2}\right) \\
d_{2}(1-\varepsilon) T\left(r, f_{2}\right) & \leq d_{2} T\left(r, f_{2}\right)+o\left(T\left(r, f_{2}\right)\right) \leq 2 \sigma_{2} \sum_{j=1}^{n_{2}} T\left(r+C, f_{1}\right)+o\left(T\left(r, f_{1}\right)\right) \\
& \leq 2 n_{2} \sigma_{2}(1+\varepsilon) T\left(r+C, f_{1}\right)
\end{aligned}\right.
$$

From (23) and for all $r \geq r_{0} \geq \frac{1}{\varepsilon}$, we have

$$
\begin{aligned}
& d_{1} d_{2}(1-\varepsilon)^{2} T\left(r, f_{1}\right) \leq 4 n_{1} n_{2} \sigma_{1} \sigma_{2}(1+\varepsilon)^{2} T\left(r+2 C, f_{1}\right) \\
& d_{1} d_{2}(1-\varepsilon)^{2} T\left(r, f_{2}\right) \leq 4 n_{1} n_{2} \sigma_{1} \sigma_{2}(1+\varepsilon)^{2} T\left(r+2 C, f_{2}\right)
\end{aligned}
$$

Iterating (24) and (25), we can get

$$
T\left(r+2 k C, f_{t}\right) \geq\left(\frac{d_{1} d_{2}}{4 n_{1} n_{2} \sigma_{1} \sigma_{2}}\left(\frac{1-\varepsilon}{1+\varepsilon}\right)^{2}\right)^{k} T\left(r, f_{t}\right), \quad t=1,2,
$$

which holds for $r \geq r_{0}$ and $k \in \mathbb{N}$. Let $k \rightarrow \infty$, for any $r \in\left[r_{0}, r_{0}+C\right)$, set $R:=r+2 k C \geq$ $r_{0}+C$, we have $k \geq \frac{R-r_{0}-C}{2 C}$. Then from (26) we can get

$$
\begin{aligned}
T\left(R, f_{t}\right) & \geq\left(\frac{d_{1} d_{2}}{4 n_{1} n_{2} \sigma_{1} \sigma_{2}}\left(\frac{1-\varepsilon}{1+\varepsilon}\right)^{2}\right)^{\frac{R-r_{0}-C}{2 C}} T\left(r_{0}, f_{t}\right) \\
& \geq K_{t}\left(\frac{d_{1} d_{2}}{4 n_{1} n_{2} \sigma_{1} \sigma_{2}}\left(\frac{1-\varepsilon}{1+\varepsilon}\right)^{2}\right)^{\frac{R}{2 C}},
\end{aligned}
$$

where

$$
K_{t}=\left(\frac{d_{1} d_{2}}{4 n_{1} n_{2} \sigma_{1} \sigma_{2}}\left(\frac{1-\varepsilon}{1+\varepsilon}\right)^{2}\right)^{\frac{-r_{0}-C}{2 C}} T\left(r_{0}, f_{t}\right), \quad t=1,2 .
$$

Thus, this completes the proof of Theorem 1.9.

\section{The Proof of Theorem 1.10}

From the assumptions of Theorem 1.10, by using the same argument as in Theorem 1.9, for any given $\varepsilon\left(0<\varepsilon<\frac{\sqrt{d_{1} d_{2}}-\sqrt{4 n_{1} n_{2} \sigma_{1} \sigma_{2}}}{\sqrt{d_{1} d_{2}}+\sqrt{4 n_{1} n_{2} \sigma_{1} \sigma_{2}}}\right)$ and all $r \geq r_{0} \geq \frac{1}{\varepsilon}$, we have

$$
\left\{\begin{aligned}
d_{1}(1-\varepsilon) T\left(r, f_{1}\right) & \leq d_{1} T\left(r, f_{1}\right)+S\left(r, f_{1}\right) \leq 2 \sigma_{1} \sum_{j=1}^{n_{1}} T\left(r+C, f_{2}\right)+S\left(r, f_{2}\right) \\
& \leq 2 n_{1} \sigma_{1}(1+\varepsilon) T\left(r+C, f_{2}\right), \\
d_{2}(1-\varepsilon) T\left(r, f_{2}\right) & \leq d_{2} T\left(r, f_{2}\right)+S\left(r, f_{2}\right) \leq 2 \sigma_{2} \sum_{j=1}^{n_{2}} T\left(r+C, f_{1}\right)+S\left(r, f_{1}\right) \\
& \leq 2 n_{2} \sigma_{2}(1+\varepsilon) T\left(r+C, f_{1}\right),
\end{aligned}\right.
$$

outside of a possible exceptional set of finite logarithmic measure.

It follows that

$$
d_{1} d_{2}(1-\varepsilon)^{2} T\left(r, f_{t}\right) \leq 4 n_{1} n_{2} \sigma_{1} \sigma_{2}(1+\varepsilon)^{2} T\left(r+2 C, f_{t}\right), \quad t=1,2,
$$


outside of a possible exceptional set of finite logarithmic measure. From (27) and Lemma 2.3, it follows that for every $\alpha>1$, there exists $r_{0}>0$ such that

$$
T\left(\alpha^{2} r+2 C, f_{t}\right) \geq \frac{d_{1} d_{2}(1-\varepsilon)^{2}}{4 n_{1} n_{2} \sigma_{1} \sigma_{2}(1+\varepsilon)^{2}} T\left(r, f_{t}\right)=: \zeta T\left(r, f_{t}\right), \quad t=1,2
$$

and $T\left(r, f_{t}\right)>1$ holds for all $r \geq r_{0}$, where $\zeta>1$. Inductively, for any positive integer $k \in \mathbb{N}$ and $r \geq r_{0}$, from (28), we have

$$
T\left(\alpha^{2 k} r+\frac{\alpha^{2 k}-1}{\alpha^{2}-1} 2 C, f_{t}\right) \geq \zeta^{k} T\left(r, f_{t}\right), \quad t=1,2 .
$$

By using the same argument as in [9, Theorem 1.11], we can get that $\mu\left(f_{t}\right)=\infty(t=1,2)$ easily.

Thus, this completes the proof of Theorem 1.10 .

Competing interests

The authors declare that they have no competing interests.

\section{Authors' contributions}

HW and HYX completed the main part of this article, HYX, HW and BXL corrected the main theorems. All authors read and approved the final manuscript.

\section{Acknowledgements}

The authors thank the referee for his/her valuable suggestions to improve the present article. This work was supported by the NSFC (61202313), the Natural Science Foundation of Jiang-Xi Province in China (Grant No. 2010GQS0119, No. 20122BAB201016, No. 20122BAB201044) and the National Science and Technology Support Plan (2012BAH25F02).

Received: 7 January 2013 Accepted: 5 March 2013 Published: 26 March 2013

\section{References}

1. Hayman, WK: Meromorphic Functions. The Clarendon Press, Oxford (1964)

2. Yang, L: Value Distribution Theory. Springer, Berlin (1993)

3. Yi, HX, Yang, CC: Uniqueness Theory of Meromorphic Functions. Kluwer Academic, Dordrecht (2003) (Chinese original: Science Press, Beijing, 1995.)

4. Shimomura, S: Entire solutions of a polynomial difference equation. J. Fac. Sci. Univ. Tokyo Sect. IA Math. 28, 253-266 (1981)

5. Yanagihara, N: Meromorphic solutions of some difference equations. Funkc. Ekvacioj 23, 309-326 (1980)

6. Ablowitz, MJ, Halburd, RG, Herbst, B: On the extension of the Painlevé property to difference equations. Nonlinearity $13,889-905(2000)$

7. Halburd, RG, Korhonen, RJ: Finite-order meromorphic solutions and the discrete Painleve equations. Proc. Lond. Math. Soc. 94, 443-474 (2007)

8. Halburd RG, R, Korhonen, RJ: Meromorphic solutions of difference equations, integrability and the discrete Painlev'e equations. J. Phys. A, Math. Theor. 40, R1-R38 (2007)

9. Heittokangas, J, Korhonen, RJ, Laine, I, Rieppo, J, Tohge, K: Complex difference equations of Malmquist type. Comput. Methods Funct. Theory 1(1), 27-39 (2001)

10. Barnett, DC, Halburd, RG, Korhonen, RJ, Morgan, W: Nevanlinna theory for the q-difference operator and meromorphic solutions of q-difference equations. Proc. R. Soc. Edinb., Sect. A, Math. 137, 457-474 (2007)

11. Bergweiler, W, Ishizaki, K, Yanagihara, N: Growth of meromorphic solutions of some functional equations I. Aequ. Math. 63(1/2), 140-151 (2002)

12. Chen, ZX: Growth and zeros of meromorphic solution of some linear difference equations. J. Math. Anal. Appl. 373, 235-241 (2011)

13. Chiang, YM, Feng, SJ: On the Nevanlinna characteristic of $f(z+\eta)$ and difference equations in the complex plane. Ramanujan J. 16, 105-129 (2008)

14. Gao, LY: On meromorphic solutions of a type of system of composite functional equations. Acta Sci. Math. 32B(2) 800-806 (2012)

15. Gao, LY: Systems of complex difference equations of Malmquist type. Acta Math. Sin. 55, 293-300 (2012)

16. Gao, LY: Estimates of $N$-function and $m$-function of meromorphic solutions of systems of complex difference equations. Acta Sci. Math. 32B(4), 1495-1502 (2012)

17. Gundersen, GG, Heittokangas, J, Laine, I, Rieppo, J, Yang, D: Meromorphic solutions of generalized Schröder equations. Aequ. Math. 63, 110-135 (2002)

18. Halburd, RG, Korhonen, RJ: Difference analogue of the lemma on the logarithmic derivative with applications to difference equations. J. Math. Anal. Appl. 314, 477-487 (2006)

19. Halburd, RG, Korhonen, RJ: Nevanlinna theory for the difference operator. Ann. Acad. Sci. Fenn., Math. 31, 463-478 (2006) 
20. Laine, I, Rieppo, J, Silvennoinen, H: Remarks on complex difference equations. Comput. Methods Funct. Theory 5(1), 77-88 (2005)

21. Laine, I: Nevanlinna Theory and Complex Differential Equations. de Gruyter, Berlin (1993)

22. Korhonen, R: A new Clunie type theorem for difference polynomials. J. Differ. Equ. Appl. 17, 387-400 (2011)

23. Silvennoinen, $\mathrm{H}$ : Meromorphic solutions of some composite functional equations. Ann. Acad. Sci. Fenn., Math. Diss. $13,14-20(2003)$

24. Wang, J: Growth and poles of meromorphic solutions of some difference equations. J. Math. Anal. Appl. 379, 367-377 (2011)

25. Zheng, XM, Chen, ZX: Some properties of meromorphic solutions of q-difference equations. J. Math. Anal. Appl. 361, 472-480 (2010)

doi:10.1186/1687-1847-2013-75

Cite this article as: Wang et al.: The poles and growth of solutions of systems of complex difference equations. Advances in Difference Equations $20132013: 75$

Submit your manuscript to a SpringerOpen ${ }^{\circ}$ journal and benefit from:

- Convenient online submission

- Rigorous peer review

- Immediate publication on acceptance

- Open access: articles freely available online

- High visibility within the field

- Retaining the copyright to your article

Submit your next manuscript at $>$ springeropen.com 